\title{
Characterizations of discrete Sugeno integrals as polynomial functions over distributive lattices
}

\author{
Miguel Couceiro \\ Mathematics Research Unit, FSTC, University of Luxembourg \\ 6, rue Coudenhove-Kalergi, L-1359 Luxembourg, Luxembourg \\ Jean-Luc Marichal ${ }^{1}$ \\ Mathematics Research Unit, FSTC, University of Luxembourg \\ 6, rue Coudenhove-Kalergi, L-1359 Luxembourg, Luxembourg
}

\begin{abstract}
We give several characterizations of discrete Sugeno integrals over bounded distributive lattices, as particular cases of lattice polynomial functions, that is, functions which can be represented in the language of bounded lattices using variables and constants. We also consider the subclass of term functions as well as the classes of symmetric polynomial functions and weighted infimum and supremum functions, and present their characterizations, accordingly. Moreover, we discuss normal form representations of these functions.
\end{abstract}

Key words: Discrete Sugeno integral, distributive lattice, lattice polynomial function, normal form, median decomposition, homogeneity, functional equation.

\section{Introduction}

We are interested in the so-called (discrete) Sugeno integral, which was introduced by Sugeno in 30,31] over real intervals and then widely investigated in aggregation theory, due to the many applications in fuzzy set theory, data fusion, decision making, pattern recognition, image analysis, etc. (for general

\footnotetext{
Email addresses: miguel.couceiro[at] uni.lu (Miguel Couceiro), jean-luc.marichal [at] uni.lu (Jean-Luc Marichal).

1 Corresponding author
} 
background, see [2,19] and for a recent reference, see [18]). In particular, it plays a relevant role as a preference functional in the qualitative frameworks of multicriteria decision making and decision making under uncertainty (for a survey, see [13]).

Another appealing feature of the Sugeno integral is that, unlike other wellestablished aggregation functions, it can be defined over ordered domains (not necessarily linearly ordered), where the usual arithmetic operations are not necessarily available. By focusing on the ordered structure of real intervals, Marichal [23] observed that Sugeno integrals can be seen as particular lattice polynomial functions, namely those that are idempotent. This fact enables us to naturally extend the original definition of the Sugeno integral to idempotent polynomial functions over bounded distributive lattices; see [24]. (Recall that a lattice polynomial function is simply a combination of projections and constant functions using the fundamental lattice operations.)

The notion of lattice polynomial function is a natural and well-established concept in lattice theory (see Birkhoff [4], Burris and Sankappanavar [5], Grätzer [20], Rudeanu [29]) and it appears in complete analogy with classical notions such as that of real polynomial functions. Indeed, just as polynomial functions of several real variables constitute the most basic functions over the field of real numbers, the lattice polynomial functions can be seen as the most elementary functions defined on an arbitrary bounded lattice. As first observed by Goodstein [17], by assuming distributivity, lattice polynomial functions become exactly those functions which can be represented in conjunctive and disjunctive normal forms.

Clearly, not every function on a bounded lattice $L$ can be represented by a lattice polynomial since such a function is necessarily nondecreasing and not every function $f: L^{n} \rightarrow L$ is nondecreasing. But even in the nondecreasing case, many fail to have such a representation (take, for instance, the real interval $[0,1]$ and consider $f:[0,1] \rightarrow[0,1]$ given by $f(x)=0$, if $x \leqslant 0.5$, and $f(x)=1$, otherwise). This fact raises the question: which nondecreasing functions constitute lattice polynomial functions?

This problem was first considered by Kindermann [21] who showed that the polynomial functions on a finite lattice are exactly those monotone functions "preserving" (compatible with) all of its tolerances. For descriptions of the latter, see Czedli and Klukovits [11] and Chajda [6].

Motivated by the aggregation theory setting, in [8] the authors took a more direct approach to polynomial functions and provided characterizations of polynomial functions on arbitrary (possibly infinite) bounded distributive lattices both as solutions of certain functional equations (such as the median decomposition system, see [24]) and in terms of necessary and sufficient con- 
ditions which have natural interpretations in the realm of decision making and aggregation theory.

One of the main purposes of the current paper is to reveal overlaps between the theories of lattice functions and aggregation functions, and present useful applications of this framework. To this extent, in this paper we provide an explanatory view of the characterizations given in [8], for instance, considering questions of independence (irredundancy) of the characterizing properties, and particularize them to the special case of Sugeno integrals. Moreover, using this connection, we study certain subclasses of polynomial functions which translate into noteworthy subclasses of Sugeno integrals.

The structure of the article is as follows. In $\S 2$, we recall the basic notions and present the preliminary results needed throughout the paper. Lattice polynomial functions are then considered in $\S 3$. We start by discussing representations of polynomial functions in normal form (such as the classical disjunctive and conjunctive normal forms) as well as the question of (non)uniqueness of these representations. This is done in $\$ 3.1$. In $\$ 3.2$, we present the various properties of polynomial functions which were used in [8] to completely describe the lattice polynomial functions. $\S 4$ is then devoted to the characterization of the subclass of Sugeno integrals as well as some other relevant subclasses, namely, of symmetric functions and of weighted infimum and supremum functions.

\section{Basic notions and terminology}

In this section we recall some notions and terminology needed throughout this paper. For further background in lattice theory we refer the reader to, e.g., Davey and Priestley [12], Grätzer [20], and Rudeanu [29].

A lattice is an algebraic structure $\langle L, \wedge, \vee\rangle$ where $L$ is a nonempty set, called universe, and where the two binary operations $\wedge$ and $\vee$ satisfy the commutative, associative, absorption, and idempotent laws. With no danger of ambiguity, we will denote a lattice by its universe. A lattice $L$ is said to be distributive if, for every $a, b, c \in L$,

$a \vee(b \wedge c)=(a \vee b) \wedge(a \vee c)$ or, equivalently, $a \wedge(b \vee c)=(a \wedge b) \vee(a \wedge c)$

Throughout this paper, we let $L$ denote an arbitrary bounded distributive lattice with least and greatest elements 0 and 1 , respectively. For $a, b \in L$, $a \leqslant b$ simply means that $a \wedge b=a$ or, equivalently, $a \vee b=b$. A chain is simply a lattice such that for every $a, b \in L$ we have $a \leqslant b$ or $b \leqslant a$. A subset $S$ of a lattice $L$ is said to be convex if for every $a, b \in S$ and every $c \in L$ such that $a \leqslant c \leqslant b$, we have $c \in S$. For any subset $S \subseteq L$, we 
denote by $\bar{S}$ the convex hull of $S$, that is, the smallest convex subset of $L$ containing $S$. For every $a, b \in S$ such that $a \leqslant b$, we denote by $[a, b]$ the interval $[a, b]=\{c \in L: a \leqslant c \leqslant b\}$. For any integer $n \geqslant 1$, we set $[n]=\{1, \ldots, n\}$.

For an arbitrary nonempty set $A$ and a lattice $L$, the set $L^{A}$ of all functions from $A$ to $L$ constitutes a lattice under the operations

$$
(f \wedge g)(x)=f(x) \wedge g(x) \quad \text { and } \quad(f \vee g)(x)=f(x) \vee g(x)
$$

for every $f, g \in L^{A}$. In particular, any lattice $L$ induces a lattice structure on the Cartesian product $L^{n}, n \geqslant 1$, by defining $\wedge$ and $\vee$ componentwise, i.e.,

$$
\begin{aligned}
& \left(a_{1}, \ldots, a_{n}\right) \wedge\left(b_{1}, \ldots, b_{n}\right)=\left(a_{1} \wedge b_{1}, \ldots, a_{n} \wedge b_{n}\right) \\
& \left(a_{1}, \ldots, a_{n}\right) \vee\left(b_{1}, \ldots, b_{n}\right)=\left(a_{1} \vee b_{1}, \ldots, a_{n} \vee b_{n}\right)
\end{aligned}
$$

We denote the elements of $L$ by lower case letters $a, b, c, \ldots$, and the elements of $L^{n}, n>1$, by bold face letters $\mathbf{a}, \mathbf{b}, \mathbf{c}, \ldots$. We also use $\mathbf{0}$ and $\mathbf{1}$ to denote the least element and greatest element, respectively, of $L^{n}$. For $c \in L$ and $\mathbf{x}=\left(x_{1}, \ldots, x_{n}\right) \in L^{n}$, set

$$
\mathbf{x} \wedge c=\left(x_{1} \wedge c, \ldots, x_{n} \wedge c\right) \text { and } \quad \mathbf{x} \vee c=\left(x_{1} \vee c, \ldots, x_{n} \vee c\right)
$$

The range of a function $f: L^{n} \rightarrow L$ is defined by $\mathcal{R}_{f}=\left\{f(\mathbf{x}): \mathbf{x} \in L^{n}\right\}$. The diagonal section of $f$ is the function $\delta_{f}: L \rightarrow L$ defined by $\delta_{f}(x)=f(x, \ldots, x)$. A function $f: L^{n} \rightarrow L$ is said to be nondecreasing (in each variable) if, for every $\mathbf{a}, \mathbf{b} \in L^{n}$ such that $\mathbf{a} \leqslant \mathbf{b}$, we have $f(\mathbf{a}) \leqslant f(\mathbf{b})$. Note that if $f$ is nondecreasing, then $\overline{\mathcal{R}}_{f}=[f(\mathbf{0}), f(\mathbf{1})]$. A function $f: L^{n} \rightarrow L$ is said to be a $\wedge$-homomorphism (resp. a $\vee$-homomorphism) if, for every $\mathbf{x}, \mathbf{y} \in L^{n}$, we have $f(\mathbf{x} \wedge \mathbf{y})=f(\mathbf{x}) \wedge f(\mathbf{y})($ resp. $f(\mathbf{x} \vee \mathbf{y})=f(\mathbf{x}) \vee f(\mathbf{y}))$.

We finish this section with the notion of lattice functions that we will be interested in hereafter. The class of lattice polynomial functions (or simply, polynomial functions) from $L^{n}$ to $L$ is defined recursively as follows:

(i) For each $i \in[n]$ and each $c \in L$, the projection $\mathbf{x} \mapsto x_{i}$ and the constant function $\mathbf{x} \mapsto c$ are polynomial functions from $L^{n}$ to $L$.

(ii) If $f$ and $g$ are polynomial functions from $L^{n}$ to $L$, then $f \vee g$ and $f \wedge g$ are polynomial functions from $L^{n}$ to $L$.

(iii) Any polynomial function from $L^{n}$ to $L$ is obtained by finitely many applications of the rules (i) and (ii).

We refer to those polynomial functions constructed from projections by finitely many applications of (ii) as lattice term functions (or simply, term functions). A well-known example of a term function is the ternary median function, which is given by 


$$
\begin{aligned}
\operatorname{median}\left(x_{1}, x_{2}, x_{3}\right) & =\left(x_{1} \wedge x_{2}\right) \vee\left(x_{2} \wedge x_{3}\right) \vee\left(x_{3} \wedge x_{1}\right) \\
& =\left(x_{1} \vee x_{2}\right) \wedge\left(x_{2} \vee x_{3}\right) \wedge\left(x_{3} \vee x_{1}\right)
\end{aligned}
$$

Fact 1 Every polynomial function $f: L^{n} \rightarrow L$ is nondecreasing.

Remark 2 Lattice polynomial functions are also referred to as lattice functions (Goodstein [17]), algebraic functions (Burris and Sankappanavar [5]), or weighted lattice polynomial functions (Marichal [24]), whereas lattice term functions are also referred to as lattice polynomials (Birkhoff [4] and Grätzer [20]).

\section{$3 \quad$ Lattice polynomial functions}

In this section, we discuss the (non)uniqueness of normal form representations of polynomial functions and present in an explanatory manner the characterization of these functions given in [8].

\subsection{Representations of polynomial functions}

Goodstein [17] showed that in the case of bounded distributive lattices, polynomial functions are exactly those which allow representations in disjunctive and conjunctive normal forms (see Proposition 3 below, first appearing in [17, Lemma 2.2]; see also Rudeanu [29, Chapter 3, §3] for a later reference). In this subsection we study such normal form representations of polynomial functions. We completely describe all possible disjunctive and conjunctive normal form representations of a given polynomial function and provide necessary and sufficient conditions which guarantee their uniqueness.

Proposition 3 Let $f: L^{n} \rightarrow L$ be a function. The following conditions are equivalent:

(i) $f$ is a polynomial function.

(ii) There exists $\alpha: 2^{[n]} \rightarrow L$ such that $f(\mathbf{x})=\bigvee_{I \subseteq[n]}\left(\alpha(I) \wedge \bigwedge_{i \in I} x_{i}\right)$.

(iii) There exists $\beta: 2^{[n]} \rightarrow L$ such that $f(\mathbf{x})=\bigwedge_{I \subseteq[n]}\left(\beta(I) \vee \bigvee_{i \in I} x_{i}\right)$

The expressions given in (ii) and (iii) of Proposition 3 are usually referred to as the disjunctive normal form (DNF) representation and the conjunctive normal form (CNF) representation, respectively, of the polynomial function $f$.

Remark 4 Proposition 3 can be easily adjusted to term functions by requiring $\alpha$ and $\beta$ to be nonconstant functions from $2^{[n]}$ to $\{0,1\}$ and satisfying $\alpha(\varnothing)=0$ and $\beta(\varnothing)=1$, respectively. 
The following corollaries belong to the folklore of lattice theory and are immediate consequences of Theorems D and E in [17].

Corollary 5 Every polynomial function is completely determined by its restriction to $\{0,1\}^{n}$.

Corollary 6 A function $g:\{0,1\}^{n} \rightarrow L$ can be extended to a polynomial function $f: L^{n} \rightarrow L$ if and only if it is nondecreasing. In this case, the extension is unique.

It is easy to see that the DNF and CNF representations of a polynomial function $f: L^{n} \rightarrow L$ are not necessarily unique. For instance, we have $x_{1} \mathrm{~V}$ $\left(x_{1} \wedge x_{2}\right)=x_{1}=x_{1} \wedge\left(x_{1} \vee x_{2}\right)$.

For each $I \subseteq[n]$, let $\mathbf{e}_{I}$ be the element of $L^{n}$ whose $i$ th component is 1 , if $i \in I$, and 0 , otherwise. Let $\alpha_{f}: 2^{[n]} \rightarrow L$ be the function given by $\alpha_{f}(I)=f\left(\mathbf{e}_{I}\right)$ and consider the function $\alpha_{f}^{*}: 2^{[n]} \rightarrow L$ defined by

$$
\alpha_{f}^{*}(I)= \begin{cases}\alpha_{f}(I), & \text { if } \bigvee_{J \varsubsetneqq I} \alpha_{f}(J)<\alpha_{f}(I), \\ 0, & \text { otherwise }\end{cases}
$$

Observe that by nondecreasing monotonicity, $\bigvee_{J \varsubsetneqq I} \alpha_{f}(J) \leqslant \alpha_{f}(I)$ for every $I \subseteq[n]$, and if $\bigvee_{J \nsubseteq I} \alpha_{f}(J)=\alpha_{f}(I)$, then

$$
\left(\alpha_{f}(I) \wedge \bigwedge_{i \in I} x_{i}\right) \vee \bigvee_{J \nsubseteq I}\left(\alpha_{f}(J) \wedge \bigwedge_{i \in J} x_{i}\right)=\bigvee_{J \nsubseteq I}\left(\alpha_{f}(J) \wedge \bigwedge_{i \in J} x_{i}\right)
$$

Thus $\alpha_{f}$ and $\alpha_{f}^{*}$ give rise to two, possibly distinct, DNF representations of $f$, i.e.,

$$
f(\mathbf{x})=\bigvee_{I \subseteq[n]}\left(\alpha_{f}(I) \wedge \bigwedge_{i \in I} x_{i}\right)=\bigvee_{I \subseteq[n]}\left(\alpha_{f}^{*}(I) \wedge \bigwedge_{i \in I} x_{i}\right)
$$

For each polynomial function $f: L^{n} \rightarrow L$, set

$$
\operatorname{DNF}(f)=\left\{\alpha \in L^{2^{[n]}}: f(\mathbf{x})=\bigvee_{I \subseteq[n]}\left(\alpha(I) \wedge \bigwedge_{i \in I} x_{i}\right)\right\}
$$

and let $\mathrm{A}(f)$ be the set of all those maps $\alpha \in L^{2^{[n]}}$ such that, for every $I \subseteq[n]$,

- $\alpha(I) \leqslant \alpha_{f}(I)$

- $\bigvee_{J \subseteq I} \alpha(J)=\alpha_{f}(I)$ whenever $\bigvee_{J \varsubsetneqq I} \alpha_{f}(J)<\alpha_{f}(I)$

Proposition 7 For any polynomial function $f: L^{n} \rightarrow L$, we have $\operatorname{DNF}(f)=$ $\mathrm{A}(f)$. 
Proof. Let $\alpha \in \mathrm{A}(f)$ and let $I \subseteq[n]$. For any $J \subseteq I$, we have $\alpha_{f}^{*}(J) \leqslant$ $\mathrm{V}_{K \subseteq I} \alpha(K)$ and hence

$$
f\left(\mathbf{e}_{I}\right)=\bigvee_{J \subseteq I} \alpha_{f}^{*}(J) \leqslant \bigvee_{J \subseteq I} \alpha(J) \leqslant \bigvee_{J \subseteq I} \alpha_{f}(J)=\alpha_{f}(I)=f\left(\mathbf{e}_{I}\right) .
$$

By Corollary 5, $\alpha \in \operatorname{DNF}(f)$ and hence $\mathrm{A}(f) \subseteq \operatorname{DNF}(f)$.

Now let $\alpha \in \operatorname{DNF}(f)$ and let $I \subseteq[n]$. By definition, $\alpha(I) \leqslant \bigvee_{J \subseteq I} \alpha(J)=$ $f\left(\mathbf{e}_{I}\right)=\alpha_{f}(I)$. It follows that $\alpha \in \mathrm{A}(f)$ and hence $\operatorname{DNF}(f) \subseteq \mathrm{A}(f)$.

Dually, Let $\beta_{f}: 2^{[n]} \rightarrow L$ be the function given by $\beta_{f}(I)=f\left(\mathbf{e}_{[n] \backslash I}\right)$ and consider the function $\beta_{f}^{*}: 2^{[n]} \rightarrow L$ defined by

$$
\beta_{f}^{*}(I)= \begin{cases}\beta_{f}(I), & \text { if } \bigwedge_{J \nsubseteq I} \beta_{f}(J)>\beta_{f}(I) \\ 1, & \text { otherwise }\end{cases}
$$

As before, both $\beta_{f}$ and $\beta_{f}^{*}$ give rise to two, possibly distinct, CNF representations of $f$. For each polynomial function $f: L^{n} \rightarrow L$, set

$$
\mathrm{CNF}(f)=\left\{\beta \in L^{2^{[n]}}: f(\mathbf{x})=\bigwedge_{I \subseteq[n]}\left(\beta(I) \vee \bigvee_{i \in I} x_{i}\right)\right\}
$$

and let $\mathrm{B}(f)$ be the set of all those maps $\beta \in L^{2^{[n]}}$ such that, for every $I \subseteq[n]$,

- $\beta(I) \geqslant \beta_{f}(I)$,

- $\bigwedge_{J \subseteq I} \beta(J)=\beta_{f}(I)$ whenever $\bigwedge_{J \nsubseteq I} \beta_{f}(J)>\beta_{f}(I)$.

In complete analogy, we have the following result, dual to Proposition 7

Proposition 8 For any polynomial function $f: L^{n} \rightarrow L$, we have $\operatorname{CNF}(f)=$ $\mathrm{B}(f)$.

Remark 9 Propositions 7 and 8 were established in [24, §3] when $L$ is a chain, and in that case it was shown that both $\mathrm{A}(f)$ and $\mathrm{B}(f)$ also constitute chains. As it is easy to verify, this property does not hold in the general case of bounded distributive lattices. For instance, $\operatorname{DNF}(f)$ is closed under $\vee$ but not necessarily under $\wedge$, and dually, $\operatorname{CNF}(f)$ is closed under $\wedge$ but not necessarily under $\vee$.

Using Propositions 7 and 8 , we obtain the following result which determines in which cases the CNF and DNF representations are unique.

Corollary 10 Let $f: L^{n} \rightarrow L$ be a polynomial function. Then $f$ has a unique $D N F$ (resp. CNF) representation if and only if for every $I \subseteq[n]$, 
(i) $\bigvee_{J \complement_{I}} \alpha_{f}(J)<\alpha_{f}(I)\left(\right.$ resp. $\left.\wedge_{J C_{I}} \beta_{f}(J)>\beta_{f}(I)\right)$, and

(ii) there is no $b \in L \backslash\left\{\alpha_{f}(I)\right\}$ (resp. $\left.c \in L \backslash\left\{\beta_{f}(I)\right\}\right)$ such that $\alpha_{f}(I)=$ $b \vee \bigvee_{J \nsubseteq I} \alpha_{f}(J)\left(\right.$ resp. $\left.\beta_{f}(I)=c \wedge \wedge_{J \nsubseteq I} \beta_{f}(J)\right)$.

Proof. We show that the result holds for the DNF representation; the other claim can be verified dually. Consider a polynomial function $f: L^{n} \rightarrow L$. Using Proposition 7 it is not difficult to verify that conditions $(i)$ and (ii) suffice to guarantee that $\operatorname{DNF}(f)$ is a singleton. Indeed, let $\alpha$ be a map in $\mathrm{DNF}(f)$ and, for the sake of a contradiction, suppose that there exists $I \subseteq[n]$ such that $\alpha(I)<\alpha_{f}(I)$. By condition $(i), \bigvee_{J \nsubseteq I} \alpha_{f}(J)<\alpha_{f}(I)$, and since there is no $b \in L \backslash\left\{\alpha_{f}(I)\right\}$ such that $\alpha_{f}(I)=b \vee \bigvee_{J \nsubseteq I} \alpha_{f}(J)$, it follows that $\alpha(I) \vee \bigvee_{J \subsetneq I} \alpha(J)<\alpha_{f}(I)$. Thus $\alpha \notin \mathrm{A}(f)$ which contradicts Proposition 7 ,

Now to see that the converse also holds, note that if for some $I \subseteq[n]$, $\bigvee_{J \nsubseteq I} \alpha_{f}(J)=\alpha_{f}(I)$, then $\alpha_{f}^{*} \neq \alpha_{f}$ and thus $f$ does not have a unique DNF representation. So assume that condition $(i)$ holds for every $I \subseteq[n]$, but suppose that for some $I^{\prime} \subseteq[n]$ there exists $b \in L \backslash\left\{\alpha_{f}\left(I^{\prime}\right)\right\}$ such that $\alpha_{f}\left(I^{\prime}\right)=b \vee \bigvee_{J \nsubseteq I^{\prime}} \alpha_{f}(J)$. Consider the map $\alpha \in L^{[[n]}$ given by $\alpha(I)=\alpha_{f}(I)$, for every $I \neq I^{\prime}$, and $\alpha\left(I^{\prime}\right)=b$ otherwise. Clearly, $\alpha \neq \alpha_{f}$ but $\alpha \in \mathrm{A}(f)$, and hence, by Proposition 7, $\alpha \in \operatorname{DNF}(f)$. Thus $f$ does not have a unique DNF representation.

Remark 11 (i) Clearly, condition (ii) of Corollary 10 is redundant when L is a chain. However this is not the case when $L$ contains two incomparable elements $a$ and $b$. For instance, by distributivity we have $((a \vee b) \wedge x) \vee a=$ $(b \wedge x) \vee a$, for every $x \in L$.

(ii) Note that $\alpha_{f}$ is the only isotone set function in $\operatorname{DNF}(f)$ and, similarly, $\beta_{f}$ is the only antitone set function in $\mathrm{CNF}(f)$.

\subsection{Properties and characterizations of polynomial functions}

In this subsection, we recall several properties of polynomial functions and discuss the relations between them. Combinations of these properties yield the various characterizations of polynomial functions presented in [8]. Throughout this subsection, let $S$ be a nonempty subset of $L$.

We say that a function $f: L^{n} \rightarrow L$ is

- $\wedge_{S}$-homogeneous if, for every $\mathbf{x} \in L^{n}$ and every $c \in S$, we have $f(\mathbf{x} \wedge c)=$ $f(\mathbf{x}) \wedge c$.

- $\vee_{S}$-homogeneous if, for every $\mathbf{x} \in L^{n}$ and every $c \in S$, we have $f(\mathbf{x} \vee c)=$ $f(\mathbf{x}) \vee c$.

- $S$-idempotent if, for every $c \in S$, we have $f(c, \ldots, c)=c$. 
For every integer $m \geqslant 1$, every vector $\mathbf{x} \in L^{m}$, and every $f: L^{n} \rightarrow L$, we define $\langle\mathbf{x}\rangle_{f} \in L^{m}$ as the $m$-tuple $\langle\mathbf{x}\rangle_{f}=\operatorname{median}(f(\mathbf{0}), \mathbf{x}, f(\mathbf{1}))$, where the right-hand side median is taken componentwise.

Proposition 12 A function $f: L^{n} \rightarrow L$ is $\wedge_{S^{-}}$and $\vee_{S^{-h o m o g e n e o u s ~ i f ~ a n d ~}}$ only if it satisfies $f(\operatorname{median}(r, \mathbf{x}, s))=\operatorname{median}(r, f(\mathbf{x}), s)$ for every $\mathbf{x} \in L^{n}$ and every $r, s \in S$. Furthermore, if $f(\mathbf{0}), f(\mathbf{1}) \in S$ and $f(\mathbf{0}) \leqslant f(\mathbf{x}) \leqslant f(\mathbf{1})$, then $f(\mathbf{x})=f\left(\langle\mathbf{x}\rangle_{f}\right)$.

Lemma 13 If $f: L^{n} \rightarrow L$ is $\wedge_{S^{-}}$and $\vee_{S^{-}}$homogeneous, then it is $S$-idempotent.

Proof. If $f$ is $\wedge_{S^{-}}$and $\vee_{S^{-}}$homogeneous, then for any $c \in S$,

$$
f(c, \ldots, c) \wedge c=f(c, \ldots, c)=f(c, \ldots, c) \vee c
$$

and thus $f$ is $S$-idempotent.

Proposition 14 ([8]) Let $f: L^{n} \rightarrow L$ be a nondecreasing function. Then

(i) If $f$ is $\wedge_{\overline{\mathcal{R}}_{f}}$ - or $\vee_{\overline{\mathcal{R}}_{f}}$-homogeneous, then it is $\overline{\mathcal{R}}_{f}$-idempotent. In particular, $\mathcal{R}_{f}=\overline{\mathcal{R}}_{f}=[f(\mathbf{0}), f(\mathbf{1})]$ and hence $f$ has a convex range.

(ii) If $f$ is a polynomial function, then it is $\wedge_{\overline{\mathcal{R}}_{f}}$ - and $\vee_{\overline{\mathcal{R}}_{f}}$-homogeneous.

We say that a function $f: L^{n} \rightarrow L$ is

- horizontally $\wedge_{S}$-decomposable if, for every $\mathbf{x} \in L^{n}$ and every $c \in S$, we have $f(\mathbf{x})=f(\mathbf{x} \vee c) \wedge f\left([\mathbf{x}]^{c}\right)$, where $[\mathbf{x}]^{c}$ is the $n$-tuple whose $i$ th component is 1 , if $x_{i} \geqslant c$, and $x_{i}$, otherwise.

- horizontally $\vee_{S}$-decomposable if, for every $\mathbf{x} \in L^{n}$ and every $c \in S$, we have $f(\mathbf{x})=f(\mathbf{x} \wedge c) \vee f\left([\mathbf{x}]_{c}\right)$, where $[\mathbf{x}]_{c}$ is the $n$-tuple whose $i$ th component is 0 , if $x_{i} \leqslant c$, and $x_{i}$, otherwise.

Remark 15 (i) In the realm of aggregation, the homogeneity properties can be interpreted as follows: Aggregating components cut at a certain level is the same as cutting at the same level the aggregated value of those components. Moreover, for $S$-idempotent functions $f: L^{n} \rightarrow L, \wedge_{S}$ - and $\vee_{S}$-homogeneity can be reformulated as $f(\mathbf{x} \wedge c)=f(\mathbf{x}) \wedge f(c, \ldots, c)$ and $f(\mathbf{x} \vee c)=f(\mathbf{x}) \vee f(c, \ldots, c)$, respectively, with $c \in S$, which reveals the "homomorphic" nature of $f$.

(ii) Horizontal $\wedge_{S}$-decomposability of a function $f: L^{n} \rightarrow L$ can be interpreted as follows: For any $\mathbf{x} \in L^{n}$ and any horizontal $\wedge_{S}$-decomposition of $\mathbf{x}$ with respect to a level $c \in S$, namely $\mathbf{x}=(\mathbf{x} \vee c) \wedge[\mathbf{x}]^{c}, f(\mathbf{x})$ decomposes with respect to $\wedge$. A similar interpretation holds for horizontal $\vee_{S}$-decomposability. 
(iii) The concepts of $\wedge_{S}$ - and $\vee_{S}$-homogeneity were used by Fodor and Roubens [16] to axiomatize certain classes of aggregation functions in the case when $S=L$ is the real interval $[0,1]$. The concept of horizontal $\vee_{S^{-}}$ decomposability was introduced, also in the case when $S=L$ is the real interval [0,1], by Benvenuti et al. [3] as a general property of the Sugeno integral, and referred to as "horizontal maxitivity".

For any $\mathbf{x}=\left(x_{1}, \ldots, x_{n}\right) \in L^{n}$, any $k \in[n]$, and any $c \in L$, set

$$
\mathbf{x}_{k}^{c}=\left(x_{1}, \ldots, x_{k-1}, c, x_{k+1}, \ldots, x_{n}\right)
$$

We say that a function $f: L^{n} \rightarrow L$ is median decomposable if, for every $\mathbf{x} \in L^{n}$, $f$ satisfies the median decomposition system

$$
f(\mathbf{x})=\operatorname{median}\left(f\left(\mathbf{x}_{k}^{0}\right), x_{k}, f\left(\mathbf{x}_{k}^{1}\right)\right) \quad(k=1, \ldots, n) .
$$

Theorem 16 ([24, Theorem 17]) The solutions of the median decomposition system (1) are exactly the polynomial functions from $L^{n}$ to $L$.

Remark 17 A comparative study of normal form representations of Boolean functions was presented in Couceiro et al. [7] where it was shown that the so-called median normal form representation, in which Boolean functions are expressed as repeated applications of the median function to variables, negated variables, and constants, provides a more efficient representation than the classical conjunctive normal form, disjunctive normal form and polynomial representations (the latter are also called Zhegalkin polynomial representations due to [32] or Reed-Muller polynomial representations due to [25,28]). Even though algorithms for converting the classical CNF, DNF, and polynomial representations into this median normal form were provided, no hint was given on how to produce median representations, e.g., from truth tables. In the case of nondecreasing functions, Theorem 16 naturally leads to a recursive procedure for obtaining median representations of functions independent from the way functions are given. Indeed, by setting an ordering of variables, say, the canonical ordering of variables, we can repeatedly apply Theorem 16 to the variables of any given function in order to derive a nested formula made of medians applied to variables and constants. By making use of tools in [7], namely the decomposition of any Boolean function as a nondecreasing function composed with variables and negated variables, this procedure can be easily extended to any Boolean function. Unfortunately, this approach seems to produce median expressions which are not optimal in the sense of [7]. To this extent one needs to find rules to simplify the median expressions produced by the above algorithm. This constitutes an interesting problem for future research.

We say that a function $f: L^{n} \rightarrow L$ is strongly idempotent if, for every $\mathbf{x} \in L^{n}$ 
and every $k \in[n]$, we have

$$
f\left(x_{1}, \ldots, x_{k-1}, f(\mathbf{x}), x_{k+1}, \ldots, x_{n}\right)=f(\mathbf{x}) .
$$

Moreover, we say that a function $f: L^{n} \rightarrow L, n>1$, has a componentwise convex range if, for every $\mathbf{a} \in L^{n}$ and every $k \in[n]$, the unary function $f_{\mathbf{a}}^{k}: L \rightarrow L$, given by $f_{\mathbf{a}}^{k}(x)=f\left(\mathbf{a}_{k}^{x}\right)$, has a convex range. As we are going to observe (see Remark 20 $(v))$, componentwise range convexity extends continuity to the realm of ordered structures.

Let $f: L^{n} \rightarrow L$ be a function and consider the following properties:

$\left(\mathbf{P}_{\wedge}\right) f$ is a componentwise $\wedge$-homomorphism, that is, $f\left(\mathbf{x}_{k}^{a \wedge b}\right)=f\left(\mathbf{x}_{k}^{a}\right) \wedge f\left(\mathbf{x}_{k}^{b}\right)$ for all $\mathrm{x} \in L^{n}, a, b \in L$, and $k \in[n]$.

$\left(\mathbf{P}_{\vee}\right) f$ is a componentwise $\vee$-homomorphism, that is, $f\left(\mathbf{x}_{k}^{a \vee b}\right)=f\left(\mathbf{x}_{k}^{a}\right) \vee f\left(\mathbf{x}_{k}^{b}\right)$ for all $\mathbf{x} \in L^{n}, a, b \in L$, and $k \in[n]$.

Fact 18 If $f: L^{n} \rightarrow L$ satisfies $\mathbf{P}_{\wedge}$ or $\mathbf{P}_{\vee}$, then it is nondecreasing.

The following result completely characterizes the class of polynomial functions in terms of the properties above.

Theorem 19 ([8]) Let $f: L^{n} \rightarrow L$ be a function. The following conditions are equivalent:

(i) $f$ is a polynomial function.

(ii) $f$ is median decomposable.

(iii) $f$ satisfies $\mathbf{P}_{\wedge}$ and $\mathbf{P}_{\vee}$, and is strongly idempotent, has a convex range and a componentwise convex range.

(iv) $f$ is nondecreasing, $\wedge_{\overline{\mathcal{R}}_{f}}$-homogeneous, and $\vee_{\overline{\mathcal{R}}_{f}}$-homogeneous.

(v) f satisfies $\mathbf{P}_{\vee}$, and is $\wedge_{\overline{\mathcal{R}}_{f}}$-homogeneous and horizontally $\vee_{\overline{\mathcal{R}}_{f}}$-decomposable.

(vi) $f$ satisfies $\mathbf{P}_{\wedge}$, and is horizontally $\wedge_{\overline{\mathcal{R}}_{f}}$-decomposable and $\vee_{\overline{\mathcal{R}}_{f}}$-homogeneous.

(vii) f satisfies $\mathbf{P}_{\wedge}$ and $\mathbf{P}_{\vee}$, and is $\overline{\mathcal{R}}_{f}$-idempotent, horizontally $\wedge \overline{\mathcal{R}}_{f}$-decomposable, and horizontally $\vee_{\overline{\mathcal{R}}_{f}}$-decomposable.

By Propositions 12 and 14, any lattice polynomial function $f: L^{n} \rightarrow L$ satisfies $f(\mathbf{x})=f\left(\langle\mathbf{x}\rangle_{f}\right)$. Using this fact, horizontal $\wedge_{\overline{\mathcal{R}}_{f}}$-decomposability (resp. horizontal $\vee_{\overline{\mathcal{R}}_{f}}$-decomposability) can be replaced with horizontal $\wedge_{L}$-decomposability (resp. horizontal $\vee_{L}$-decomposability) in the assertions $(v)$ - $(v i i)$ of Theorem [19.

Remark 20 (i) In the case when $L$ is a chain, the conditions $\mathbf{P}_{\wedge}$ and $\mathbf{P}_{\vee}$ can be replaced in Theorem 19 by nondecreasing monotonicity. In this case, the properties involved in the characterization given in (iii) do not make use of the lattice operations. Further relaxations to the conditions of Theorem 19 can be found in [9]. 
(ii) Consider the conditions given in assertion (iii) of Theorem 19. (a) The unary function $f(x)=x^{2}$ on the real interval $L=[0,1]$ satisfies all these conditions but strong idempotency. (b) Let $L=\{0, a, 1\} \times\{0,1\}$, and consider $f: L \rightarrow L$ given by $f(11)=f(10)=f(a 1)=a 1$ and $f(00)=$ $f(a 0)=f(01)=01$. This function satisfies all the conditions except $\mathbf{P}_{\wedge}$ and $\mathbf{P}_{\vee}$. (c) Let $L=\{0, a, 1\}$ and consider the function $f: L^{2} \rightarrow L$ given by

$$
f\left(x_{1}, x_{2}\right)= \begin{cases}0, & \text { if } 0 \in\left\{x_{1}, x_{2}\right\}, \\ a, & \text { if } x_{1}=a \text { and } x_{2} \geqslant a, \\ 1, & \text { if } x_{1}=1 \text { and } x_{2} \geqslant a .\end{cases}
$$

This function satisfies all the conditions except that it does not have a componentwise convex range.

(iii) Any Boolean function $f:\{0,1\}^{n} \rightarrow\{0,1\}$ satisfying $f(\mathbf{0}) \leqslant f(\mathbf{x}) \leqslant f(\mathbf{1})$ is $\wedge_{\overline{\mathcal{R}}_{f}}$-homogeneous, $\vee_{\overline{\mathcal{R}}_{f}}$-homogeneous, horizontally $\wedge_{\overline{\mathcal{R}}_{f}}$-decomposable, and horizontally $\vee_{\overline{\mathcal{R}}_{f}}$-decomposable. Moreover, as soon as $n \geqslant 3$, there are such $\overline{\mathcal{R}}_{f}$-idempotent Boolean functions which are not nondecreasing, thus showing that nondecreasing monotonicity and the properties $\mathbf{P}_{\wedge}$ and $\mathbf{P}_{\vee}$ (which reduce to nondecreasing monotonicity in the case of chains) are necessary in assertions (iv)-(vii) of Theorem 19 .

(iv) The set $\overline{\mathcal{R}}_{f}$ cannot be replaced by $\mathcal{R}_{f}$ in the assertions (iv)-(vii) of Theorem 19 . To see this, let $L=\{0,1 / 3,2 / 3,1\}$ with the canonical ordering of the elements and define $f: L \rightarrow L$ by

$$
f(x)= \begin{cases}0, & \text { if } x \leqslant 1 / 3 \\ 1, & \text { otherwise }\end{cases}
$$

Clearly, $f$ is $\wedge_{\mathcal{R}_{f}}$-homogeneous, $\vee_{\mathcal{R}_{f}}$-homogeneous, horizontally $\wedge_{\mathcal{R}_{f}}$-decomposable, and horizontally $\vee_{\mathcal{R}_{f}}$-decomposable, but it is not median decomposable.

(v) In the special case of real interval lattices, i.e., where $L=[a, b]$ for reals $a \leqslant b$, the property of having a convex range, as well as the property of having a componentwise convex range, are consequences of continuity. More precisely, for nondecreasing functions $f:[a, b]^{n} \rightarrow \mathbb{R}$, being continuous is equivalent to being continuous in each variable, and this latter property is equivalent to having a componentwise convex range. In fact, since polynomial functions are continuous, the conditions of having a convex range and a componentwise convex range can be replaced by continuity in (iii) of Theorem 19. Also, by Proposition 14, in each of the conditions (iv)-(vii) we can add continuity and replace $\overline{\mathcal{R}}_{f}$ by $\mathcal{R}_{f}$. 


\section{Some particular classes of lattice polynomial functions}

We now consider some important subclasses of polynomial functions, namely, those of (discrete) Sugeno integrals, of symmetric polynomial functions, and of weighted infimum and supremum functions, and provide their characterizations, accordingly.

\subsection{Discrete Sugeno integrals}

A function $f: L^{n} \rightarrow L$ is said to be idempotent if it is $L$-idempotent.

Fact 21 A polynomial function is $\{0,1\}$-idempotent if and only if it is idempotent.

In [24, §4], \{0,1\}-idempotent polynomial functions are referred to as (discrete) Sugeno integrals. They coincide exactly with those functions $\mathcal{S}_{\mu}: L^{n} \rightarrow L$ for which there is a fuzzy measure $\mu$ such that

$$
\mathcal{S}_{\mu}(\mathbf{x})=\bigvee_{I \subseteq[n]}\left(\mu(I) \wedge \bigwedge_{i \in I} x_{i}\right)
$$

Here, by a fuzzy measure $\mu$, we simply mean a set function $\mu: 2^{[n]} \rightarrow L$ satisfying $\mu(I) \leqslant \mu\left(I^{\prime}\right)$ whenever $I \subseteq I^{\prime}$, and $\mu(\varnothing)=0$ and $\mu([n])=1$.

Proposition 22 ([24, Proposition 12]) For any polynomial function $f: L^{n} \rightarrow$ $L$ there is a fuzzy measure $\mu: 2^{[n]} \rightarrow L$ such that $f(\mathbf{x})=\operatorname{median}\left(f(\mathbf{0}), \mathcal{S}_{\mu}(\mathbf{x}), f(\mathbf{1})\right)=$ $\left\langle\mathcal{S}_{\mu}(\mathbf{x})\right\rangle_{f}$.

From Lemma 13, Theorem 19 (iv), and Fact 21, we immediately obtain the following characterization of the Sugeno integrals, which was previously established in the case of real variables in [22, Theorem 4.2].

Corollary 23 A function $f: L^{n} \rightarrow L$ is a Sugeno integral if and only if it is nondecreasing, $\wedge_{L}$-homogeneous, and $\vee_{L}$-homogeneous.

Even though Corollary 23 can be derived from condition (iv) of Theorem 19 by simply modifying the two homogeneity properties, to proceed similarly with conditions $(v)$ and $(v i)$, it is necessary to add the conditions of $\{1\}$ idempotency and $\{0\}$-idempotency, respectively. To see this, let $L$ be a chain with at least three elements and consider the unary functions $f(x)=x \wedge d$ and $f^{\prime}(x)=x \vee d$, where $d \in L \backslash\{0,1\}$. Clearly, $f$ is $\wedge_{L}$-homogeneous and

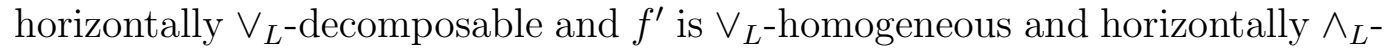
decomposable. However, neither $f$ nor $f^{\prime}$ is a Sugeno integral. To see that these 
additions are sufficient, just note that $\wedge_{L}$-homogeneity (resp. $\vee_{L}$-homogeneity) implies $\{0\}$-idempotency (resp. $\{1\}$-idempotency).

Theorem 24 Let $f: L^{n} \rightarrow L$ be a function. The following conditions are equivalent:

(i) $f$ is a Sugeno integral.

(ii) $f$ is $\{0,1\}$-idempotent and median decomposable.

(iii) $f$ satisfies $\mathbf{P}_{\wedge}$ and $\mathbf{P}_{\vee}$, and is strongly idempotent, has range $L$ and a componentwise convex range.

(iv) $f$ is nondecreasing, $\wedge_{L}$-homogeneous, and $\vee_{L}$-homogeneous.

(v) $f$ satisfies $\mathbf{P}_{\vee}$, and is $\{1\}$-idempotent, $\wedge_{L}$-homogeneous, and horizontally $\vee_{L}$-decomposable.

(vi) $f$ satisfies $\mathbf{P}_{\wedge}$, and is $\{0\}$-idempotent, horizontally $\wedge_{L}$-decomposable, and $\vee_{L}$-homogeneous.

(vii) $f$ satisfies $\mathbf{P}_{\wedge}$ and $\mathbf{P}_{\vee}$, and is L-idempotent, horizontally $\wedge_{L}$-decomposable, and horizontally $\vee_{L}$-decomposable.

\subsection{Lattice term functions}

A function $f: L^{n} \rightarrow L$ is said to be conservative if, for every $\mathbf{x} \in L^{n}$, we have

$$
f(\mathbf{x}) \in\left\{x_{1}, \ldots, x_{n}\right\}
$$

Remark 25 Condition (2) was used in the binary case by Pouzet et al. [27].

Clearly, every conservative function is idempotent. Furthermore, if $L$ is a chain, then every term function $f: L^{n} \rightarrow L$ is conservative. This is not necessarily true in the general case of bounded distributive lattices. To see this, let $L=$ $\{0, a, b, 1\}$, where $a \vee b=1$ and $a \wedge b=0$, and let $f\left(x_{1}, x_{2}\right)=x_{1} \vee x_{2}$. Obviously, $f(a, b) \notin\{a, b\}$.

Thus, in order to characterize term functions in the general case of bounded distributive lattices, we need to relax conservativeness. We say that a function $f: L^{n} \rightarrow L$ is weakly conservative if (2) holds for every $\mathbf{x} \in\{0,1\}^{n}$. By definition, every weakly conservative function is $\{0,1\}$-idempotent.

Corollary 26 Let $f: L^{n} \rightarrow L$ be a Sugeno integral. Then $f$ is a term function if and only if it is weakly conservative. 


\subsection{Symmetric polynomial functions}

An important property of functions is that of symmetry which basically translates into saying that each argument of the function has the same "weight" on the values of the function. Formally, a function $f: L^{n} \rightarrow L$ is symmetric if, for every permutation $\sigma$ on $[n]$, we have $f\left(x_{1}, \ldots, x_{n}\right)=f\left(x_{\sigma(1)}, \ldots, x_{\sigma(n)}\right)$.

Noteworthy examples of symmetric functions are the so-called order statistic functions; see for instance Ovchinnikov [26]. For any arity $n \geqslant 1$ and any $k \in[n]$, the $k$ th order statistic function is the term function $\operatorname{os}_{k}: L^{n} \rightarrow L$ defined by

$$
\operatorname{Os}_{k}(\mathbf{x})=\bigvee_{\substack{I \subseteq[n] \\|I|=n-k+1}} \bigwedge_{i \in I} x_{i}=\bigwedge_{\substack{I \subseteq[n] \\|I|=k}} \bigvee_{i \in I} x_{i}
$$

As a matter of convenience, we set $\mathrm{os}_{0}=0$ and $\mathrm{os}_{n+1}=1$. It is easy to verify that a polynomial function $f: L^{n} \rightarrow L$ is symmetric if and only if the set function $\alpha_{f}: 2^{[n]} \rightarrow L$, given by $\alpha_{f}(I)=f\left(\mathbf{e}_{I}\right)$, is cardinality based, that is, for every $I, I^{\prime} \subseteq[n]$, if $|I|=\left|I^{\prime}\right|$, then $\alpha_{f}(I)=\alpha_{f}\left(I^{\prime}\right)$. Thus, by letting $w_{f}:\{0, \ldots, n\} \rightarrow L$ be the isotone function satisfying $\alpha_{f}(I)=w_{f}(|I|)$ for every $I \subseteq[n]$, we get

$$
\begin{aligned}
f(\mathbf{x}) & =\bigvee_{I \subseteq[n]}\left(\alpha_{f}(I) \wedge \bigwedge_{i \in I} x_{i}\right)=\bigvee_{k=0}^{n}\left(w_{f}(k) \wedge \bigvee_{\substack{I \subseteq[n] \\
|I|=k}} \bigwedge_{i \in I} x_{i}\right) \\
& =\bigvee_{k=0}^{n}\left(w_{f}(k) \wedge \mathrm{OS}_{n-k+1}(\mathbf{x})\right) .
\end{aligned}
$$

We can make dual observations on the CNF representation of symmetric polynomial functions. Indeed, a polynomial function $f: L^{n} \rightarrow L$ is symmetric if and only if the set function $\beta_{f}: 2^{[n]} \rightarrow L$, given by $\beta_{f}(I)=f\left(\mathbf{e}_{[n] \backslash I}\right)$, is cardinality based. Thus, by letting $v_{f}:\{0, \ldots, n\} \rightarrow L$ be the antitone function satisfying $\beta_{f}(I)=v_{f}(|I|)$ for every $I \subseteq[n]$, we get

$$
\begin{aligned}
f(\mathbf{x}) & =\bigwedge_{I \subseteq[n]}\left(\beta_{f}(I) \vee \bigvee_{i \in I} x_{i}\right)=\bigwedge_{k=0}^{n}\left(v_{f}(k) \vee \bigwedge_{\substack{I \subseteq[n] \\
|I|=k}} \bigvee_{i \in I} x_{i}\right) \\
& =\bigwedge_{k=0}^{n}\left(v_{f}(k) \vee \operatorname{Os}_{k}(\mathbf{x})\right) .
\end{aligned}
$$

Moreover, we have that $v_{f}(i)=w_{f}(n-i)$ for all $i \in\{0, \ldots, n\}$.

These observations are reassembled in the following result characterizing those 
polynomial functions which are symmetric. The equivalence between conditions $(i),(i i)$, and (iii) was observed in [15, Proposition 9].

Theorem 27 Let $f: L^{n} \rightarrow L$ be a polynomial function. Then the following conditions are equivalent:

(i) $f$ is symmetric,

(ii) there is a cardinality based function $\alpha: 2^{[n]} \rightarrow L$ such that

$$
f(\mathbf{x})=\bigvee_{I \subseteq[n]}\left(\alpha(I) \wedge \bigwedge_{i \in I} x_{i}\right)
$$

(iii) there is an isotone function $w:\{0, \ldots, n\} \rightarrow L$ such that

$$
f(\mathbf{x})=\bigvee_{k=0}^{n}\left(w(n-k) \wedge \operatorname{os}_{k+1}(\mathbf{x})\right)
$$

(iv) there is a cardinality based function $\beta: 2^{[n]} \rightarrow L$ such that

$$
f(\mathbf{x})=\bigwedge_{I \subseteq[n]}\left(\beta(I) \vee \bigvee_{i \in I} x_{i}\right)
$$

(v) there is an antitone function $v:\{0, \ldots, n\} \rightarrow L$ such that

$$
f(\mathbf{x})=\bigwedge_{k=0}^{n}\left(v(k) \vee \mathrm{os}_{k}(\mathbf{x})\right)
$$

For every $n \geqslant 1$, the $(2 n+1)$-ary median function is defined by (see [1, Chapter IV])

$$
\operatorname{median}_{2 n+1}(\mathbf{x})=\operatorname{os}_{n+1}(\mathbf{x})=\bigvee_{\substack{I \subseteq[2 n+1] \\|I|=n+1}} \bigwedge_{i \in I} x_{i}=\bigwedge_{\substack{I \subseteq[2 n+1] \\|I|=n+1}} \bigvee_{i \in I} x_{i}
$$

Corollary 28 (i) A polynomial function $f: L^{n} \rightarrow L$ is symmetric if and only if there exists an isotone (or, equivalently, antitone) function $w:\{0, \ldots, n\} \rightarrow$ $L$ such that

$$
f\left(x_{1}, \ldots, x_{n}\right)=\operatorname{median}_{2 n+1}\left(x_{1}, \ldots, x_{n}, w(0), \ldots, w(n)\right) .
$$

(ii) A Sugeno integral $f: L^{n} \rightarrow L$ is symmetric if and only if there exists an isotone (or, equivalently, antitone) function $w:\{1, \ldots, n-1\} \rightarrow L$ such that

$$
f\left(x_{1}, \ldots, x_{n}\right)=\operatorname{median}_{2 n-1}\left(x_{1}, \ldots, x_{n}, w(1), \ldots, w(n-1)\right) .
$$

(iii) A term function is symmetric if and only if it is an order statistic. 
Proof. To see that $(i)$ holds, just note that the function $f: L^{n} \rightarrow L$ given by (3) is a symmetric polynomial function. The converse follows from (iii) of Theorem 27. The statements $(i i)$ and $($ iii $)$ are consequences of $(i)$.

\subsection{Weighted infimum and supremum functions}

We say that a function $f: L^{n} \rightarrow L$ is a weighted infimum function if there are $w_{0}, w_{1}, \ldots, w_{n} \in L$ such that

$$
f(\mathbf{x})=w_{0} \wedge \bigwedge_{i \in[n]}\left(w_{i} \vee x_{i}\right)
$$

Similarly, we say that $f: L^{n} \rightarrow L$ is a weighted supremum function if there are $v_{0}, v_{1}, \ldots, v_{n} \in L$ such that

$$
f(\mathbf{x})=v_{0} \vee \bigvee_{i \in[n]}\left(v_{i} \wedge x_{i}\right)
$$

Note that the weights $w_{0}$ and $v_{0}$ simply constitute the maximum and the minimum possible values of the function, respectively.

Proposition 29 If $f: L^{n} \rightarrow L$ is a $\wedge$-homomorphism or a $\vee$-homomorphism, then it is nondecreasing. Furthermore, ^-homomorphicity (resp. $\vee$-homomorphicity) implies horizontal $\wedge$-decomposability (resp. horizontal $\vee$-decomposability).

Theorem 30 Let $f: L^{n} \rightarrow L$ be a polynomial function. Then

(i) $f$ is a weighted infimum function if and only if it is a $\wedge$-homomorphism.

(ii) $f$ is a weighted supremum function if and only if it is a $\vee$-homomorphism.

Proof. We prove $(i)$. The proof of $(i i)$ follows similarly. Suppose first that $f$ is a weighted infimum function. Then, by distributivity we have that for every $\mathbf{x}, \mathbf{y} \in L^{n}$,

$$
\begin{aligned}
f(\mathbf{x} \wedge \mathbf{y}) & =w_{0} \wedge \bigwedge_{i \in[n]}\left(w_{i} \vee\left(x_{i} \wedge y_{i}\right)\right)=w_{0} \wedge \bigwedge_{i \in[n]}\left(\left(w_{i} \vee x_{i}\right) \wedge\left(w_{i} \vee y_{i}\right)\right) \\
& =\left(w_{0} \wedge \bigwedge_{i \in[n]}\left(w_{i} \vee x_{i}\right)\right) \wedge\left(w_{0} \wedge \bigwedge_{i \in[n]}\left(w_{i} \vee x_{i}\right)\right) \\
& =f(\mathbf{x}) \wedge f(\mathbf{y}) .
\end{aligned}
$$

In other words, $f$ is a $\wedge$-homomorphism. 
Now we show that if $f$ is a $\wedge$-homomorphism, then it is a weighted infimum function. Observe first that, as a polynomial function, $f$ is $\wedge_{\overline{\mathcal{R}}_{f}}{ }^{-}$and $\vee_{\overline{\mathcal{R}}_{f}}{ }^{-}$ homogeneous, and thus, by Proposition [12, for every $i \in[n]$ and every $c \in L$,

$$
f\left(\mathbf{1}_{i}^{0} \vee c\right)=f\left(\left\langle\mathbf{1}_{i}^{0} \vee c\right\rangle_{f}\right)=f\left(\left\langle\mathbf{1}_{i}^{0}\right\rangle_{f}\right) \vee\langle c\rangle_{f}=f\left(\mathbf{1}_{i}^{0}\right) \vee\langle c\rangle_{f}
$$

Therefore, by $\wedge$-homomorphicity we have

$$
\begin{aligned}
f(\mathbf{x}) & =f\left(\bigwedge_{i \in[n]}\left(\mathbf{1}_{i}^{0} \vee x_{i}\right)\right)=\bigwedge_{i \in[n]}\left(f\left(\mathbf{1}_{i}^{0}\right) \vee\left\langle x_{i}\right\rangle_{f}\right) \\
& =\bigwedge_{i \in[n]}\left(f\left(\mathbf{1}_{i}^{0}\right) \vee\left(f(\mathbf{1}) \wedge\left(x_{i} \vee f(\mathbf{0})\right)\right)\right) \\
& =f(\mathbf{1}) \wedge \bigwedge_{i \in[n]}\left(f\left(\mathbf{1}_{i}^{0}\right) \vee x_{i}\right) .
\end{aligned}
$$

Setting $w_{0}=f(\mathbf{1})$ and $w_{i}=f\left(\mathbf{1}_{i}^{0}\right)$ for $i \in[n]$, we have that $f$ is a weighted infimum function.

Remark 31 (i) Idempotent weighted infimum functions $f: L^{n} \rightarrow L$ are those functions (4) for which $w_{0}=1$ and $\wedge_{i \in[n]} w_{i}=0$. Dually, idempotent weighted supremum functions $f: L^{n} \rightarrow L$ are those functions (5) for which $v_{0}=0$ and $\vee_{i \in[n]} v_{i}=1$. These functions were introduced on real intervals by Dubois and Prade [14] in fuzzy set theory, and referred to as "weighted minimum and maximum functions".

(ii) The restriction of Theorem 30 to idempotent functions (Sugeno integrals) was already established in the special case of real intervals in [22, Theorem 5.2].

\section{Conclusion and future work}

In this paper we have discussed discrete Sugeno integrals when regarded as particular lattice polynomial functions, namely idempotent polynomial functions. Having this definition in mind, we have investigated normal form representations of polynomial functions and provided complete descriptions of the set of all disjunctive and conjunctive normal forms of a given function, and obtained necessary and sufficient conditions which guarantee uniqueness of such representations. Then, using the characterizations of polynomial functions given in terms of well-established properties in aggregation theory (here generalized to ordered domains where incomparabilities may occur), we presented various axiomatizations for the class of Sugeno integrals over bounded distributive lattices. Furthermore, we discussed redundancy issues concerning the set of 
axioms and showed that their independence in most cases. Regarding the axiomatization given in Theorem 19 (iii), it remains open whether or not range convexity is redundant under the other conditions.

Some noteworthy subclasses of Sugeno integrals were also considered, namely those of

- term functions, which were shown to constitute weakly conservative Sugeno integrals,

- symmetric Sugeno integrals, which, as in the case of real closed intervals, were shown to constitute Sugeno integrals determined by cardinality based capacities or in fact median functions up to fixing arguments, and

- weighted infimum and weighted supremum functions, which naturally extend the so-called weighted minimum and weighted maximum functions and which were shown to constitute $\wedge$ - and $\vee$-semilattice homomorphisms.

Concerning directions of further research, the authors have proposed within this framework, a very natural generalization to the Sugeno integral, namely that of quasi-Sugeno integral [10] and which falls within the scope of utility based decision making. More precisely, these are functions $f: L^{n} \rightarrow L$ which can be factorized as a composition

$$
f\left(x_{1}, \ldots, x_{n}\right)=p\left(\varphi\left(x_{1}\right), \ldots, \varphi\left(x_{n}\right)\right),
$$

where $p: L^{n} \rightarrow L$ is a Sugeno integral (or equivalently, a polynomial function) and $\varphi: L \rightarrow L$ a nondecreasing function (utility function). There are several extensions available within this framework, for instance, one could consider possibly different domain and codomain bounded distributive lattices $X$ and $Y$, and remove the monotonicity condition on the inner function $\varphi: X \rightarrow Y$.

\section{References}

[1] M. Barbut and B. Monjardet. Ordre et classification: algèbre et combinatoire. Tome I. Librairie Hachette, Paris, 1970.

[2] G. Beliakov, A. Pradera, and T. Calvo. Aggregation Functions: A Guide for Practitioners. Studies in Fuziness and Soft Computing. Springer, Berlin, 2007.

[3] P. Benvenuti, R. Mesiar, and D. Vivona. Monotone set functions-based integrals. In Handbook of measure theory, Vol. I, II, pages 1329-1379. NorthHolland, Amsterdam, 2002.

[4] G. Birkhoff. Lattice Theory. Third edition. American Mathematical Society Colloquium Publications, Vol. XXV. American Mathematical Society, Providence, R.I., 1967. 
[5] S. Burris and H. P. Sankappanavar. A Course in Universal Algebra, volume 78 of Graduate Texts in Mathematics. Springer-Verlag, New York, 1981.

[6] I. Chajda. Albebraic Theory of Tolerance Relations. ISBN 80-7067-042-8, Olomouc, 1991.

[7] M. Couceiro, S. Foldes, and E. Lehtonen. Composition of Post classes and normal forms of Boolean functions. Discrete Math., 306(24):3223-3243, 2006.

[8] M. Couceiro and J.-L. Marichal. Polynomial functions over bounded distributive lattices. http://arxiv.org/abs/0901.4888

[9] M. Couceiro and J.-L. Marichal. Representations and characterizations of polynomial functions on chains. J. of Multiple-Valued Logic and Soft Computing, to appear.

[10] M. Couceiro and J.-L. Marichal. Axiomatizations of quasi-polynomial functions on bounded chains. Aequationes Mathematicae, to appear.

[11] G. Czédli and L. Klukovits. A note on tolerances of idempotent algebras. Glasnik Matematički, 18(38):35-38, 1983.

[12] B. A. Davey and H. A. Priestley. Introduction to Lattices and Order. Cambridge University Press, New York, second edition, 2002.

[13] D. Dubois, J.-L. Marichal, H. Prade, M. Roubens, and R. Sabbadin. The use of the discrete Sugeno integral in decision making: a survey. Int. J. of Uncertainty, Fuzziness and Knowledge-Based Systems, 9(5):539-561, 2001.

[14] D. Dubois and H. Prade. Weighted minimum and maximum operations in fuzzy set theory. Inform. Sci., 39(2):205-210, 1986.

[15] A. Dukhovny and J.-L. Marichal. System reliability and weighted lattice polynomials. Probability in the Engineering and Informational Sciences, $22(3): 373-388,2008$.

[16] J. C. Fodor and M. Roubens. Characterization of weighted maximum and some related operations. Inform. Sci., 84(3-4):173-180, 1995.

[17] R. L. Goodstein. The solution of equations in a lattice. Proc. Roy. Soc. Edinburgh Sect. A, 67:231-242, 1965/1967.

[18] M. Grabisch, J.-L. Marichal, R. Mesiar, and E. Pap. Aggregation Functions. Encyclopedia of Mathematics and its Applications. Cambridge University Press, Cambridge, UK, 2009.

[19] M. Grabisch, T. Murofushi, and M. Sugeno, editors. Fuzzy measures and integrals, volume 40 of Studies in Fuzziness and Soft Computing. PhysicaVerlag, Heidelberg, 2000. Theory and applications.

[20] G. Grätzer. General Lattice Theory. Birkhäuser Verlag, Berlin, 2003. Second edition. 
[21] M. Kindermann. Über die Äquivalenz von Ordnungspolynomvollständigkeit und Toleranzeinfachheit endlicher Verbände. Contributions to General Algebra (Proceedings of the Klagenfurt conference 1978), 145-149, 1979.

[22] J.-L. Marichal. On Sugeno integral as an aggregation function. Fuzzy Sets and Systems, 114(3):347-365, 2000.

[23] J.-L. Marichal. An axiomatic approach of the discrete Sugeno integral as a tool to aggregate interacting criteria in a qualitative framework. IEEE Transactions on Fuzzy Systems, 9(1):164-172, 2001.

[24] J.-L. Marichal. Weighted lattice polynomials. Discrete Mathematics, $309(4): 814-820,2009$.

[25] D. E. Muller. Application of boolean algebra to switching circuit design and to error correction. IRE Trans. Electron. Comput., 3(3):6-12, 1954.

[26] S. Ovchinnikov. Means on ordered sets. Math. Social Sci., 32(1):39-56, 1996.

[27] M. Pouzet, I. G. Rosenberg, and M. G. Stone. A projection property. Algebra Universalis, 36(2):159-184, 1996.

[28] I. S. Reed. A class of multiple-error-correcting codes and the decoding scheme. IEEE Trans. Inform. Theory, 4(4):38-49, 1954.

[29] S. Rudeanu. Lattice Functions and Equations. Springer Series in Discrete Mathematics and Theoretical Computer Science. Springer-Verlag London Ltd., London, 2001.

[30] M. Sugeno. Theory of fuzzy integrals and its applications. PhD thesis, Tokyo Institute of Technology, Tokyo, 1974.

[31] M. Sugeno. Fuzzy measures and fuzzy integrals - a survey. In M. M. Gupta, G. N. Saridis, and B. R. Gaines, editors, Fuzzy automata and decision processes, pages 89-102. North-Holland, New York, 1977.

[32] I. I. Zhegalkin. On the calculation of propositions in symbolic logic. Mat. Sb., 34:9-28, 1927. (in Russian). 